\title{
Non-thermal radio emission in Wolf-Rayet stars: is binarity a pre-requisite?
}

\author{
Sean M. Dougherty \\ University of Calgary/Dominion Radio Astrophysical Observatory NRC, \\ P.O. Box 248, Penticton, B.C., Canada V2A 6 K3
}

\begin{abstract}
Radio observations of Wolf-Rayet stars currently available in the literature are examined to determine whether binarity is a common feature of WR systems with non-thermal emission. Among 24 stars with observed spectral index values, seven are definite non-thermal emitters, and six others possibly have composite thermal/non-thermal spectra. Stellar companions have been identified in $71 \%$ of the non-thermal emitters, strongly supporting a link between non-thermal emission and binarity.
\end{abstract}

\section{Discussion}

Radio emission from stellar winds is characterised by free-free continuum emission with $T_{B} \simeq 10^{4} \mathrm{~K}$ and spectral indices $\alpha \simeq+0.6$. A number of WR stars have radio properties that differ from this typical picture, with $T_{B} \simeq 10^{6-7} \mathrm{~K}$, and flat or negative spectral indices, characteristics of non-thermal synchrotron emission. In the cases of WR 146 and 147, two stellar components and the non-thermal emission region have been spatially resolved and the origin of the non-thermal emission in a stellar wind collision region is well-supported (Dougherty et al. 1996; Williams et al. 1997; Neimela et al. 1998).

To determine whether binarity is common among WR stars that exhibit non-thermal radio emission, radio spectral indices have been determined for WR stars that have been observed at more than one radio wavelength. The complete sample of stars used in this study is shown in Table 1. Sources where $\alpha$ is not significantly flatter than +0.6 are identified as thermal and those with $\alpha$ significantly lower than 0.0 are identified as non-thermal. In the case that the radio emission from a WR system is a composite of non-thermal and a thermal emission, the spectral index could be between +0.0 and +0.6 . These sources are identified as thermal/non-thermal.

Among the 24 sources in Table 1, there are 7 sources that are definite nonthermal emitters: WR $14,48,105,125,140,146$, and 147 , corresponding to $29 \%$ of the sample. Including the sources that we identify as having composite spectra gives either a total of $8(33 \%)$ or $13(54 \%)$ of the total sample, dependent on whether you chose the 3 or $2 \sigma$ significance criteria. Of the 7 definite non-thermal emitters, 5 of the systems are known binaries or multiple systems $(71 \%)$, with only WR 14 and WR 105 not being identified as such. Among the 6 sources that may have composite spectra, only two (WR 39 and 90) are identified as single stars in the 7th Catalogue of Galactic Wolf-Rayet Stars (van der Hucht 1999). Thus, of the 13 stars that exhibit or possibly exhibit non-thermal emission, 9 
Table 1. Radio spectral indices of Wolf-Rayet stars

\begin{tabular}{|c|c|c|c|c|c|c|}
\hline \multirow[t]{2}{*}{ WR } & \multirow[t]{2}{*}{ spectral type ${ }^{(9)}$} & \multirow[t]{2}{*}{ period } & \multirow[t]{2}{*}{ radio $\alpha$} & \multicolumn{2}{|c|}{ emission type } & \multirow[t]{2}{*}{ ref. } \\
\hline & & & & $3 \sigma^{a}$ & $2 \sigma^{a}$ & \\
\hline 6 & WN4b & & $+0.7 \pm 0.2$ & $\mathrm{~T}$ & $\mathrm{~T}$ & 3 \\
\hline 9 & WC5+O7 & $14.3 \mathrm{~d}$ & $>+1.7$ & $\mathrm{~T}$ & $\mathrm{~T}$ & 1 \\
\hline 11 & WC8+08.5III & $78.5 \mathrm{~d}$ & $\begin{array}{l}+0.33 \pm 0.04 \\
+0.69 \pm 0.02\end{array}$ & $\begin{array}{l}\mathrm{T} / \mathrm{NT} \\
\mathrm{T}\end{array}$ & $\begin{array}{l}\mathrm{T} / \mathrm{NT} \\
\mathrm{T}\end{array}$ & $\begin{array}{l}1 \\
4\end{array}$ \\
\hline 14 & WC7 & \multirow{5}{*}{$80.4 d$} & $<-0.7$ & NT & NT & 1 \\
\hline 16 & WN8h & & $+0.62 \pm 0.15$ & $\mathrm{~T}$ & $\mathrm{~T}$ & 2 \\
\hline 22 & WN7ha+O7.5 & & $+0.14 \pm 0.19$ & $\mathrm{~T}$ & $\mathrm{~T} / \mathrm{NT}$ & 2 \\
\hline 24 & WN6ha & & $>+1.7$ & $\mathrm{~T}$ & $\mathrm{~T}$ & 2 \\
\hline 39 & WC7 & & $-0.37 \pm 0.34$ & $\mathrm{~T}$ & $\mathrm{~T} / \mathrm{NT}$ & 1 \\
\hline 40 & WN8h & \multirow{3}{*}{$18.3 \mathrm{~d}$} & $+0.68 \pm 0.12$ & $\mathrm{~T}$ & $\mathrm{~T}^{\prime}$ & 2 \\
\hline 48 & WC6+O9.5+? & & $-0.39 \pm 0.15$ & NT & NT & 1 \\
\hline 78 & WN7h & & $+0.5 \pm 0.1$ & $\mathrm{~T}$ & $\mathrm{~T}$ & 3 \\
\hline 86 & $\mathrm{WC} 7+\mathrm{OB}^{(10)}$ & \multirow[t]{5}{*}{ Visual } & $+0.18 \pm 0.20$ & $\mathrm{~T}$ & $\mathrm{~T} / \mathrm{NT}$ & 8 \\
\hline 89 & WN8h+a & & $+0.74 \pm 0.18$ & $\mathrm{~T}$ & $\mathrm{~T}^{\prime}$ & 2 \\
\hline 90 & WC7 & & $+0.02 \pm 0.24$ & $\mathrm{~T}$ & $\mathrm{~T} / \mathrm{NT}$ & 1 \\
\hline 105 & WN9h & & $-0.27 \pm 0.09$ & NT & NT & 1 \\
\hline 112 & WC9 & & $>0.95$ & $\mathrm{~T}$ & $\mathrm{~T}$ & 1 \\
\hline 125 & WC7+O9 & \multirow[t]{3}{*}{$>15 y \mathrm{r}$} & $-0.5 \rightarrow+0.7$ & NT\&T & NT\&T & 3,7 \\
\hline 134 & WN6b & & $+0.9 \pm 0.2$ & $\mathrm{~T}$ & $\mathrm{~T}$ & 3 \\
\hline 136 & WN6b & & $+0.7 \pm 0.2$ & $\mathrm{~T}$ & $\mathrm{~T}$ & 3 \\
\hline 137 & WC7+OB & $>13 y r$ & $+0.0 \pm 0.2$ & $\mathrm{~T}$ & $\mathrm{~T} / \mathrm{NT}$ & 7 \\
\hline 140 & $\mathrm{WC} 7+\mathrm{O} 4-5$ & $7.9 \mathrm{yr}$ & $-0.5 \rightarrow+0.7$ & NT\&T & N'T\&T & 11,12 \\
\hline 145 & $\mathrm{WN} / \mathrm{C}+\mathrm{OB} ?$ & $22.5 \mathrm{~d}$ & $+0.8 \pm 0.3$ & $\mathrm{~T}$ & $\mathrm{~T}$ & 3 \\
\hline \multirow[t]{2}{*}{146} & \multirow[t]{2}{*}{$\mathrm{WC} 6+\mathrm{O}^{(5)}$} & \multirow[t]{2}{*}{ Visual } & $-0.62 \pm 0.04$ & NT & NT & 5 \\
\hline & & & $+0.74 \pm 0.20$ & $\mathrm{~T}$ & $\mathrm{~T}$ & 5 \\
\hline \multirow[t]{2}{*}{147} & \multirow[t]{2}{*}{ WN8+OB } & \multirow[t]{2}{*}{ Visual } & $-0.37 \pm 0.07$ & NT & NT & 6 \\
\hline & & & $+0.66 \pm 0.02$ & $\mathrm{~T}$ & $\mathrm{~T}$ & 6 \\
\hline
\end{tabular}

Notes: (a) Significance of difference of $\alpha$ from +0.6 .

References: (1) Leitherer et al. (1997); (2) Leitherer et al. (1995). Values of $\alpha$ calculated from radiometry listed in Table 1 of each of these papers; (3) Abbott et al. (1986); (4) Williams et al. (1990b); (5) Dougherty \& Williams (these Proceedings); (6) Williams et al. (1997); (7) Williams, private communication; (8) Dougherty et al. , in preparation; (9) van der Hucht (1999); (10) Neimela et al. (1998); (11) Williams et al. (1990a); and (12) White \& Becker (1995).

(70\%) are binary systems. This provides compelling evidence that non-thermal emission and binarity are, in some manner, connected.

\section{References}

Leitherer, C., Chapman, J.S., Koribalski, B. 1995, ApJ 450, 289

Leitherer, C., Chapman, J.S., Koribalski, B. 1997, ApJ 481, 898

Neimela, V.S., Shara, M.M., Wallace, D.J., Zurek, D.R., Moffat, A.F.J. 1998, AJ 115, 2047

van der Hucht, K.A. 1999, The 7th Catalogue of Galactic Wolf-Rayet Stars, in preparation

Williams, P.M., van der Hucht, K.A., Pollock, A.M.T., Florkowski, D.R., van der Woerd, H., Wamsteker, W.M. 1990a, MNRAS 243, 662

Williams, P.M., van der Hucht, K.A., Sandell, G., Thé, P.S., 1990b, MNRAS 244, 101

Williams, P.M., Dougherty, S.M., Davis, R.J. van der Hucht, K.A., Bode, M.F., Setia Gunawan, D.Y.A. 1997, MNRAS 289, 10 